\title{
CREACIÓN, CONSERVACIÓN Y GESTIÓN DEL PAISAJE UN ELEMENTO CLAVE PARA EL DESARROLLO RURAL EN ANDALUCÍA
}

Andreas HILDENBRAND SCHEID *

\section{INTRODUCCIÓN}

La "producción de paisaje de calidad" se considera en el documento "Bases para un Plan de Desarrollo Rural Andaluz" (PDRA, presentado en marzo de 1993 en Córdoba por el Presidente de la Comunidad Autónoma de Andalucía), como una de las nuevas funciones que la sociedad demanda al medio rural ${ }^{1}$. Por otra parte, la reciente reforma de la PAC significa que las futuras actuaciones del Gobierno andaluz en materia del paisaje rural podrán contar con un "viento favorable" desde Bruselas, ya que el Reglamento CEE núm. 2078/92 permite aprovechar recursos comunitarios para la financiación de programas de producción agraria compatibles con la protección, conservación y mejora del paisaje.

En este contexto la presente comunicación sostiene que un paisaje de calidad constituye un recurso y elemento clave para el desarrollo rural en Andalucía. Entendemos por calidad de paisaje los valores estético-visuales que residen en la manifestación formal o fisonómica del territorio, sin perder de vista, por ello, los otros valores (ecológicos, culturales, etc.) que se suelen atribuir al concepto del paisaje.

Partiendo de una exposición de las razones que fundamentan el papel clave del recurso paisaje para el desarrollo rural y de un diagnóstico de la degradación actual del paisaje rural andaluz, formulamos una serie de propuestas para posibles líneas de actuación de una política para el paisaje rural andaluz en el marco del futuro PDRA. Las propuestas hacen referencia a experiencias existentes en

* Asesor Técnico en el Servicio de Estudios y Estadísticas de la Consejería de Agricultura y Pesca de la Junta de Andalucía.

1. Consejería de Agricultura y Pesca 1993. Bases para un Plan de Desartollo Rural (PDRA), Junta de Andalucía. 
otros países que podrían orientar la elaboración e implementación de esta política en Andalucía. Se concentran en las actividades del sector FAO, siendo consciente que la calidad del paisaje rural no sólo está en manos del agricultor sino depende también de una adecuada integración en el paisaje de las actividades no agrarias.

\section{2. ¿POR QUÉ EL PAISAJE ES UN ELEMENTO CLAVE EN EL DESARROLLO RURAL ANDALUZ?}

Desde un planteamiento pluridimensional del concepto del desarrollo, que no reduce su significado a lo económico, sino persigue objetivos económicos, sociales, ecológicos y culturales, existen suficientes razones para considerar el paisaje un elemento clave para el desarrollo rural.

El medio rural hoy en día tiene que ofertar paisaje de calidad para una población urbana cada vez más amplia que desarrolla en el campo diversas actividades individuales o colectivas: el simple descanso, contemplando la "belleza" del paisaje y disfrutando sensaciones de paz y tranquilidad, el recreo y el deporte o el gran número de otras actividades relacionadas con el agroturismo. Todas estas actividades son de importancia económica, por ello, el paisaje puede ser entendido como un recurso, capaz de generar empleo y renta, y por tanto, vital para fijar la población en el campo ${ }^{2}$. Además, en la "sociedad modema de tiempo libre" estas actividades tienen una importancia creciente, por lo cual la demanda por paisaje de calidad es cada vez mayor. Pero el paisaje no sólo constituye un recurso imprescindible para eI desarrollo rural desde el punto de vista económico y demográfico sino también lo es desde el punto de vista de la salud o del bienestar físico y psíquico de los ciudadanos. Muchas de las actividades mencionadas los ciudadanos las emprenden para mantener su salud, siendo el paisaje para ellos no un mero soporte físico o espacio suficientemente libre y abierto para el ejercicio de tales actividades sino que el paisaje les ofrece también cualidades estéticas que pueden producir en el hombre a través de la percepción subjetiva multisensorial (la vista, pero también los demás sentidos) del paisaje una serie de vivencias y sensaciones positivas. Estas también juegan, como indican las investigaciones de la ecología humana, psicología ambiental y teoría estética, un importante papel para el equilibrio y bienestar psicofísico del hombre ${ }^{3}$.

La destrucción o degradación de las propiedades visuales de un paisaje en una determinada zona puede provocar un cambio en su valoración por los habitantes o visitantes que comienzan a trasladar a otros sitios su residencia o el desarrollo 
de las actividades mencionadas con la consiguiente pérdida de empleo y renta en aquella zona. Si en los proyectos de los parques tecnológicos o de las nuevas urbanizaciones turísticas se nota una creciente preocupación por la calidad estética-visual del paisaje que las empresas y los turistas tienen cada vez más en cuenta en sus decisiones de localización y viajes es conveniente que en la planificación rural se parta del mismo criterio si no se quiere poner en peligro las oportunidades para un desarrollo centrado en la movilización del potencial endógeno de recursos locales, a los cuales pertenece también el paisaje. En este contexto tampoco se debe olvidar que en muchas zonas rurales de Andalucía, agrícolamente improductivas o cuyas producciones ya no son rentables, la riqueza y calidad de sus ecosistemas y paisajes constituyen casi el único recurso aprovechable para que su población pueda superar su situación de subdesarrollo y marginalidad.

Desde el punto de vista ecológico, la conservación de determinados elementos del paisaje rural como setos, bosquetes, la vegetación de riberas o cercas y paredes de piedras no sólo mantiene la diversidad paisajística sino que conservan al mismo tiempo importantes hábitats para la fauna y flora.

El mantenimiento de superficies cultivadas por razones paisajísticas muchas veces ofrece a la sociedad la protección contra la erosión, avenidas e incendios, riesgos que cobran una especial importancia en el área mediterránea.

Por último, no se debe olvidar la importancia del paisaje como patrimonio cultural y punto de referencia para la identidad social y territorial; en Andalucía, por ejemplo, los paisajes de los olivares, de los policultivos mediterráneos y de las dehesas.

\section{LA DEGRADACIÓN DEL PAISAJE RURAL ANDALUZ}

En muchas zonas del medio rural andaluz se observa una creciente degradación del paisaje por los impactos visuales de las actividades agrarias y no agrarias.

Respecto a las actividades agrarias un diagnóstico sintético ha de constatar una pérdida de diversidad y un aumento de la monotonía así como una creciente artificialización y banalización del paisaje agrario.

Los cultivos forzados de la agricultura intensiva en el Campo de Dalias y el litoral de Huelva aplican en sus sistemas de cultivo grandes cantidades de plásticos. Así los invernaderos generan en muchos sitios una gran uniformidad, repetitividad y monotonía del paisaje por "empaquetar" bajo plástico enormes extensiones de terrenos. Al mismo tiempo suponen una intrusión visual conside- 
rable por el alto grado de visibilidad abierta o prominente de las áreas transformadas (laderas, vegas, llanuras). La introducción masiva de estructuras artificiales crea un paisaje con escasos rasgos de naturalidad y los desechos de plástico contaminan visualmente estas áreas. Las repoblaciones forestales del pasado en la Sierra Morena occidental condujeron a profundas transformaciones de la estructura del suelo (desbroces, aterrazamientos) para albergar especies no autóctonas (eucalipto) poco compatibles con el medio. Respecto a su entorno provocaron frecuentemente una notable dominancia por la escala de la actuación y un fuerte contraste visual debido a las formas geométricas con líneas rectas de la nueva cubierta vegetal que no se adaptan a la existente configuración (silueta, curvas) del terreno.

La eliminación de enclaves de árboles aislados en medio de grandes superficies de cultivos redujo en muchos sitios la diversidad del paisaje y aumentó su monotonía. La paulatina desaparición de las cercas de piedra en las dehesas priva a este paisaje de un elemento típico. El patrimonio arquitectónico de la agricultura andaluza tradicional (molinos de aceite, haciendas de olivar, etc.) sufre, en general, un deterioro. Con frecuencia los elementos construidos agrarios (silos, secaderos, etc.) no se ajustan en su diseño (volumen, tamaño, color, forma, etc.) al entorno o se convierten por su emplazamiento en elementos perturbadores de vistas o no deseables puntos focales de atracción visual. En el caso de no pocos pueblos se percibe desde determinados puntos de vista panorámicas y desde las vías de comunicación de acceso una alteración de su imagen exterior originado por actividades agrarias y no agrarias. Las manifestaciones de ella son la perturbación de vistas a la fachada histórica del pueblo, la ruptura de la silueta por edificios y a lo largo de las vías de acceso la exposición a la vista de escombros, basura, sitios de recogida de chatarra de coches y largas secuencias de invernaderos.

Otro problema en este contexto es la desaparición de los ruedos, antiguamente ocupados por huertas y multicultivos intensivos, y su transformación en un paisaje fragmentado, incoherente y heteróclito debido al abandono de tierras (barbecho social) y la actividad urbanizadora.

La degradación del paisaje no siempre se debe a un exceso de la actividad agraria sino a veces también al abandono de dicha actividad. Ejemplos son la matorralización de un gran número de dehesas (pérdida del contraste cromático entre pastos verdes y arboleda, aumento de incendios) y el abandono del cultivo en terrazas o de la ganadería y silvicultura en muchas áreas de montaña, que genera visualmente un paisaje desolador y descuidado y aumenta el riesgo de la erosión del suelo. 


\section{LÍNEAS DE ACTUACIÓN PARA UNA POLÍTICA DE CREACIÓN, CONSERVACIÓN Y GESTIÓN DEL PAISAJE RURAL EN ANDALUCÍA}

"Leitmotiv" de la política para el paisaje rural andaluz debe ser, a nuestro juicio, la opción a favor de un enfoque activo y creativo. Este consiste en que las actuaciones ejecutadas por las Administraciones Públicas o las que ellas encarguen a terceros por contratos contemplen desde el primer momento y en todo el territorio la integración de los aspectos paisajísticos, abandonando un enfoque pasivo y reactivo, hoy en día dominante, que se limita a la corrección a posteriori de los impactos negativos sobre el paisaje y a planteamientos puramente conservacionistas para unos pocos enclaves del territorio. Conservar lo que es digno de ser preservado, restaurar los paisajes degradados, emplazar y diseñar desde el principio en armonía con el paisaje existente y tener la imaginación y el valor para innovaciones que contribuyan a mejorar la potencialidad y atractividad visual de paisajes estéticamente pobres son, a nuestro juicio, los cuatro pilares fundamentales de esta política activa y creativa para el paisaje rural.

Partiendo de este enfoque, las siguientes líneas de actuación se refieren primordialmente a la dimensión paisajística de las actividades del sector FAO. Por ello contemplan sobre todo propuestas que afectan el ámbito de actuación de la Consejería de Agricultura y Pesca en la puesta en práctica del futuro PDRA. Conforme al reparto competencial en las materias FAO, ordenación del territorio, urbanismo, patrimonio histórico y turismo, se dará en algunas de estas líneas de actuación la cooperación entre ella y otras Consejerías y la Administración Local, sin olvidar la necesaria colaboración con los agentes económicos y sociales.

Por otra parte, la calidad visual del paisaje rural andaluz no se consigue solamente con la adopción de criterios paisajísticos en el seno de las políticas públicas sectoriales. Como enseña la experiencia internacional comparada, hace falta una política general para el paisaje en su conjunto desde la ordenación del territorio, que sea capaz de tener una visión general, sinóptica y plurisectorial del desarrollo paisajístico que se pretende conseguir para todo el territorio. En este sentido, la implementación práctica en el futuro PDRA de las líneas de actuación propuestas habrá de asumir y desarrollar los objetivos y directrices que podrían establecerse en los futuros planes de ordenación del territorio de nivel subregional previstos por la Ley de Ordenación del Territorio de la Comunidad Autónoma de Andalucía, aprobada en el Parlamento Andaluz.

Identificar, describir y evaluar la calidad visual existente de los paisajes agrarios andaluces y evaluar, en base a cuencas visuales y aplicando técnicas de simulación visual, los posibles impactos visuales sobre el paisaje que se derivan de los proyectos $F A O$. 
En este tema existe una rica experiencia en los Estados Unidos, tanto a nivel de la investigación sobre posibles metodologías como a nivel de la práctica administrativa ${ }^{4}$.

En España es, sobre todo, la Cátedra de Planificación y Proyectos de la ETSI Montes de Madrid quien conecta con estas experiencias. Respecto a los paisajes rurales y proyectos del sector FAO son de interés los sistemas de gestión de los recursos visuales (visual resource management system) aplicado por el Forest Service y el Soil Conservation Service del Ministerio de Agricultura de los Estados Unidos. Los rasgos comunes de estos sistemas y del que se`aplica por el Bureau of Land Management del Ministerio del lnterior son: 1) subsistemas para el inventario y la evaluación de la calidad visual de los componentes configuradores físico-bióticos del paisaje, 2) subsistemas para evaluar el uso, la visibilidad y las valoraciones del paisaje por el público, 3) la elaboración de cartografía de estos factores a fin de obtener áreas clasificadas para determinados objetivos de gestión o la formulación de niveles de calidad visual y de prioridades para la realización de intervenciones sobre el paisaje. Aprovechando las experiencias que existen en los países anglosajones, pero también en Alemania y Francia, han de elaborarse enfoques y métodos adaptados a las características específicas del paisaje rural andaluz.

Establecer criterios paisajísticos para la localización, el trazado y el diseño de los proyectos FAO.

La evaluación de la calidad visual existente en un determinado paisaje agrario y la de los posibles impactos de una actuación sobre ella culmina en la formulación de criterios y medidas que permiten evitar (evaluación preventiva), mitigar o compensar estos impactos. Lo más deseable es el enfoque de la evaluación preventiva, es decir, adoptar en los proyectos desde el principio el criterio paisajístico. En este sentido sería conveniente que la Consejería de Agricultura elabore un manual par el "buen diseño paisajístico de proyectos FAO".

4. U.S.D. of Interior (Bureau of Land Management) 1980; Visual resource management program, Washington, DC; U.S.D. of Agriculture (Forest Service) 1974; National forest landscape management, vol, 2 chapter I: The visual management system, Washing ton,; U.S.D. of Agriculture (Soil Conservation Service) 1978: Procedure to establish priorities in landscape architecture, Washington DC: Smardon, $R$. C. et al 1986;; Foundation for visual project analysis, John Wiley \& Sons, New York; Smardon, R.C. I Karp, J. 1993; The legal landscape. Guidelines for regulation environmental and aesthetic quality, Van Nostrand Reinhold (KY); Schauman, S. 1986; Countryside landscape visual assessment, en : Smardon et al, p. 103-114; Ramos, A. et al 1984: Guía para la elaboración de estudios del medio físico MOPU/ CEOTMA, Madrid; Bundesforschungsanstalt für Naturschutz und Landschaftsökologie (ed.) 1991; Landschafsbild-Eingriff-Ausgleich, Bonn. 
Los manuales anglosajones y franceses sobre repoblaciones forestales ${ }^{5}$ recomiendan por ejemplo: 1) evitar las formas demasiado geométricas, abandonando las líneas rectas, y buscar la discontinuidad de las líneas, apoyándose en las estructuras del paisaje existente y teniendo en cuenta la configuración del terreno, 2) escoger especies del entomo y conseguir una transición suave con la zona no repoblada, manteniendo un esparcimiento mayor entre las plantas de repoblación situadas en los bordes, 3) evitar los límites perpendiculares o paralelos a las curvas de nivel, 4) mantener un porcentaje mínimo de cultivos y pastos como elemento de discontinuidad (diversidad paisajística, prevención de incendios, etc.). Respecto a la localización y distribución de los invemaderos se aconseja en un estudio de la Consejería de Obras Públicas "tener en cuenta las relaciones de viabilidad, observando, por ejemplo, los siguientes criterios: 1) evitar una distribución anárquica, dando preferencia a una agrupación ordenada en unidades y polígonos, 2) localización preferente en áreas llanas y de cotas bajas, evitando su implantación en laderas y en las inmediaciones de las vías de comunicación, salvo que elementos intermedios existentes eviten su visibilidad, 3) en ausencia de éstos últimos, se puede plantar vegetación arbórea en la franja de separación entre vías de comunicación e invernaderos como instrumento de ocultación visual. En manuales suizos y alemanes sobre las consecuencias estético-visuales de medidas de la reordenación parcelaria ${ }^{7}$ se recomienda para la creación de caminos rurales, por ejemplo: 1) evitar los trazados rectilíneos dando preferencia a un trazado curvado, 2) emplear en las obras para sostener taludes tecnologías blandas (p.e. gaviones o muros de piedras secas) que se adapten al paisaje, 3) estructurar el paisaje mediante la plantación de árboles, arbustos o setos en tramos discontinuos a lo largo de los bordes y la creación de bosquetes que pueden indicar los cruces de caminos. Las características del diseño (forma, volumen, color, materiales, estilo, etc.) de edificios e instalaciones, agrícola-ganaderas han de respetar el entomo existente. Si no se pueden lograr una ubicación de baja visibilidad o una integración adecuada en el paisaje mediante el diseño, deben emplearse técnicas de ocultación o de camuflaje (mimetismo).

5. Cemagref 1981: Reboisement et paysage. For èt-loisirs et equipement de plein air, Nouvelle Serie, París; Hough y otros 1982: Design guidelines for forest management, Ministry of Natural Resources, Toronto, Ontario; MOPU 1990: Guías metodológicas para la elaboración de estudios de impacto ambiental. 3. Repoblaciones forestales, Madrid.

6. Consejeria de Obras Públicas y Transportes/CETU 1988; Estrategias para la regeneración de los paisajes litorales, Junta de Andalucía, Sevilla.

7. Office Fédéral des Forets/Office Fédéral de l'Agriculture 1983; Protection de la nature et du paysage lors d'ameliorations foncières. Guide et recomendations, Berne; Wöbse, H.H. 1984. Erlebniswirksamkeit des Landschaft und Flurbereinigung Untersuchungen zur Landeschaftsästhetik, en : Landschaft und Stadt 16, (1/2,33-54) Bayerisches Staatsministerium für Ernährung, Landwirtschaft und Forsten 1987: Lamdschaftsästhetik in der Flurbereinigung, München. 
Formular y poner en práctica programas agroambientales que contemplan la conservación y mejora del paisaje.

Estos programas pueden acogerse a la financiación que la CEE ofrece a través de su Reglamento 2078/92 y desarrollarán con medidas concretas dos principios previstos para el futuro PDRA: "la producción de paisaje de calidad" y el principio "el que conserva, cobra". La formulación de estos programas puede inspirarse en el programa MEKA ("Compensación por mercado y Conservación de] Paisaje") de 1993 del Ministerio de Agricultura del Land Baden-Württemberg en Alemania y en el programa "Countryside Stewardship", administrado por la Countryside Commission con el apoyo del Ministerio de Agricultura de Inglaterra ${ }^{8}$. Otro punto de referencia constituye el programa ROL-RAL para la conservación de elementos paisajísticos específicos del Ministerio de Agricultura de Holanda.

Como primera área de aplicación en Andalucía de este tipo de programas, que ofrecen en el marco de contratos voluntarios a los agricultores ayudas financieras por su labor como conservadores y gestores del paisaje rural, podrían elegirse las dehesas por el extraordinario valor ecológico y estético-visual de su paisaje, estudiando la posibilidad de presentar ante la CEE un programa conjunto con la Comunidad Autónoma vecina de Extremadura.

Elaborar un inventario de los paisajes agrarios tradicionales de Andalucía y formular objetivos y medidas para su conservación y gestión.

Andalucía pertenece a las regiones con una gran riqueza de este tipo de paisajes actualmente amenazados que hace necesario la puesta en práctica de medidas de protección, conservación y gestión.

El inventario podría recoger y completar por otros los 61 Paisajes Agrícolas Singulares que han sido delimitados en los Catálogos de los Planes Especiales de Protección del Medio Físico (PEPMF) aprobados por la Consejería de Obras Públicas y Transportes para cada una de las ocho provincias andaluzas. Asimismo pueden ser desarrollados y completados por las recomendaciones de gestión (p. e. control de tala, desbroce de matorral, preservación de la red de acequias) de estos planes. Aquellos paisajes agrarios tradicionales que destacan por su singularidad, sus valores estético-visuales u otros valores (ecológicos, arquitectónicos, etc.) podrían recibir algún otro tratamiento legal específico que el que ofrecen los PEPMF. En Italia existe la figura de los paisajes agrarios de interés histórico-

8. Land Baden-Württemberg (Ministerium für Ländlichen, Raum, Ernähnung, Landwitschaft und Forsten) 1993; Marjtentlastungs -und Kulturlandschaftsausgleich (MEKA; Richtlinie vom 19.1. 1993; Countryside Commission y otros 1992; Handbook for Countryside Stewardship, Cheltenham; Countryside Commission 1993; Faying for a beautiful countryside Cheltenham. 
cultural, para los cuales las regiones formulan en sus planes paisajísticos (p.e. Marche) y en sus planes de ordenación del territorio (p.e. el Veneto) una serie de prescripciones o directivas. En los demás podrían ser suficientes los incentivos para los agricultores para la conservación de elementos paisajísticos típicos (terrazas, setos, etc.). Para esta tarea es un modelo útil el programa MEKA y la amplia gama de programas del Reino Unido (Countryside Stewardship, Hedgerow Incentive Scheme, Environmentally Sensitive Areas, etc.). El Countryside Stewardship ofrece incluso un manual de gestión específico para los "historic landscapes". También los Ministerios de Agricultura y de Ordenación del Territorio de Alemania (sobre todo Baviera) prestan mucha atención al paisaje agrario tradicional mediante incentivos y directrices de ordenación en el marco de planes paisajísticos que son parte integral de los planes de ordenación del territorio. En lo que afecta a las vías agropecuarias $(30.951 \mathrm{~km}$.), un patrimonio importante en el paisaje rural tradicional, se recomienda que la recuperación y la ordenación de estos terrenos de dominio público incluyan medidas de mejora paisajísticas que podrían favorecer el uso recreativo o agroturístico de estas vías, por ejemplo, mediante la reforestación de los bordes de determinadas vías con árboles en tramos discontinuos (paseo alternando sol y sombra, disfrute de vistas al campo abierto, etc.).

Emprender medidas para conservar y mejorar la imagen de los núcleos rurales andaluces.

A la mejora de la imagen exterior de los pueblos podrá contribuir el programa de mantenimiento y restauración de los ruedos de los pueblos previsto en el documento Bases para el futuro PDRA. Al mismo objetivo servirían también medidas como la ocultación de invernaderos por la plantación de árboles a lo largo de las vías de acceso o la aplicación de técnicas de ocultación o camuflaje (mimetismo) a edificios o instalaciones agrícola-ganaderas, sin olvidar las posibilidades de un traslado, para acabar con la perturbación de vistas importantes a la fachada exterior de un pueblo. También se debe prestar atención a la imagen interior de los pueblos procurando que el emplazamiento y las características del diseño de estos edificios o instalaciones logren una integración armónica en el entorno existente. Merecen un estudio las experiencias de los Ministerios de Agricultura de los Länder alemanes que en sus programas multisectoriales para el desarrollo y la renovación de los pueblos (Dorferneuerung) prevén medidas para la conservación y mejora de la imagen exterior de los pueblos ${ }^{9}$. En Suiza la Federación aprobó un Reglamento que recoge un inventario de todas las imáge-

9. Bundesminister für Ernährung, Landwirtschaft und Forsten 1989: Dorferneurerung, MünsterHilstrup. 
nes de pueblos dignos de protección, y los Cantones aprobaron inventarios semejantes previendo además en sus planes de ordenación del territorio criterios y medidas para la protección y conservación de la imagen de los pueblos. Por otra parte la Oficina Federal de Agricultura recomienda en su manual "Protección de la naturaleza y del paisaje en las mejoras agrícolas" muchas medidas en relación con este objetivo, por ejemplo: 1) incorporar los silos en el interior de los edificios, 2) ocultar los silos exteriores mediante su emplazamiento detrás de edificios grandes o la plantación de vegetación arbórea, 3) construir un grupo de silos pequeños en vez de uno aislado de grandes dimensiones, 4) seleccionar los materiales de construcción y los colores en función del entorno y renunciar a inscripciones publicitarias visibles desde lejos ${ }^{10}$.

Sensibilizar y asesorar a los agricultores sobre su responsabilidad y utilidad económica, ecológica, social y cultural como productores y conservadores del paisaje.

En este campo convienen las siguientes medidas: 1) la celebración de jornadas y cursos de formación, 2) proyectos piloto de excelencia paisajística, es decir explotaciones modélicas desde el punto de vista paisajístico que ejercerían un importante efecto de demostración, 3) la edición de folletos de y manuales que informan sobre las técnicas agrícola-ganaderas y de construcción que contribuyen a la conservación y creación de un paisaje de calidad. Como puntos de orientación pueden servir los manuales de los programas agroambientales ingleses y los manuales suizos, uno de ellos con el título sugestivo "La naturaleza en las manos de los agricultores" ".

10. Ver 7 .

11. Office Féderal de 1'Environnement, Des Forèts et du Paysage 1992: La nature aus mains des paysans. Aménagement du territoire agricole. Beme. 